\title{
Identificando clústers culturales en el centro de Monterrey. Un análisis de caso.
}

Identifying cultural clusters in Monterrey city center. A case study.

Recibido: Junio 2018

Aceptado: Febrero 2019
Fabiola Garza-Rodríguez ${ }^{1}$

\section{Resumen}

La cultura como herramienta de regeneración urbana se utiliza desde hace varias décadas, dirigir la revitalización de centros urbanos y barrios degradados. Si bien existe una gran cantidad de estudios que investigan el fenómeno, hace falta ampliar el debate hacia la etapa del diagnóstico, específicamente hacia el concepto del mapeo cultural como herramienta de análisis urbano. Tomando como caso de estudio el centro de Monterrey, el artículo examina las manifestaciones de cultura en el territorio a partir de la identificación de recursos culturales en el tejido urbano. Se utiliza un método mixto que conjunta datos cuantitativos con métodos cualitativos de análisis como valoraciones de actores clave, observación directa e investigación documental. Los resultados muestran que el centro cuenta con un rico tejido cultural dentro de su trama urbana, el cual debido a la proximidad entre unos y otros permite identificar un clúster cultural en donde suceden dinámicas distintas al resto del territorio. Esta investigación contribuye en mostrar alternativas sobre como analizar la cultura en la ciudad. En específico, el trabajo contribuye en el uso y entendimiento de la técnica de mapeo cultural como herramienta de diagnosis e instrumento de análisis en un contexto latinoamericano.

\section{Palabras Clave:}

Regeneración urbana, Mapeo cultural, Cluster cultural, Centro.

\begin{abstract}
The use of culture as a tool of urban regeneration is not recent, since several decades ago, it has been used as a preferred concept to direct the revitalization of urban centers. Although there are large number of studies that research the phenomenon, it is necessary to broaden the debate towards the diagnostic stage, specifically towards the cultural mapping as a tool of analysis. Taking as a case study the center of Monterrey, located at northeast Mexico, the article examines culture in the territory through the identification of cultural resources in the urban center. We use a mixed method that combines quantitative data with qualitative methods of analysis like key actors narratives, direct observation and documentary research. Results show that the center has a rich cultural presence within its urban plot, which due to the proximity between each other allows us to identify the existence of a cultural cluster where dynamics that are different from the rest of the territory happen. This research contributes to show alternatives on how to analyze culture in urban geography, specially through the use of cultural mapping in a Latin American context.
\end{abstract}

\section{Keywords:}

Urban regeneration, Cultural mapping, Cultural clusters, Center.

\footnotetext{
${ }^{1}$ Nacionalidad: mexicana; adscripción: Instituto Universitario de Investigación en Ciencia y Tecnologías de la Sostenibilidad en la Universitat Politècnica de Catalunya, España; doctora en sostenibilidad. Correo electrónico: fabiola.grz@gmail.com
} 


\section{Introducción.}

En una época en donde se ha estudiado de manera extensa el fenómeno urbano desde diversas disciplinas es necesario volver a los elementos básicos para buscar nuevas respuestas para las necesidades actuales. La cultura, por ejemplo, es uno de estos elementos por moldear la sociedad y por tanto, las ciudades. El carácter holístico del concepto la hace aplicable a todas las áreas del desarrollo pudiendo ser utilizada como herramienta de desarrollo y planeación urbana (Hawkes, 2001; García, 2004; Miles y Paddison, 2005). Donde su objetivo va desde mejorar la imagen urbana hasta optimizar las condiciones y calidad de vida de los habitantes (Bianchini y Parkinson, 1993; Dinardi, 2012).

No es reciente utilizar la cultura para promover el desarrollo sostenible urbano y por tanto, regeneraciones urbanas (Bianchini y Parkinson, 1993; Hawkes, 2001; García, 2004; Miles y Paddisson, 2005). Pero visibilizar los valores y recursos culturales que existen en un determinado lugar a partir de su reconocimiento para impulsar el desarrollo local (Tavano Blessi et al., 2012; Soini y Birkeland, 2014; Duxbury et al., 2016) es la aportación que hoy en día produce.

La evolución hacia economías orientadas a servicios sitúa a la cultura como motor de desarrollo urbano. Se ha dejado de entender a la cultura como solo arte y patrimonio para considerarla un activo económico que genera nuevas oportunidades, negocios y profesionistas en la ciudad (Zukin, 1995) sirviendo a su vez como herramienta de regeneración urbana.

El interés de esta investigación nace de analizar la geografía de la cultura en lo urbano enfocado en un contexto latinoamericano: la ciudad de Monterrey. Ubicada al noreste de México, Monterrey es una metrópolis dinámica, global y vibrante que se desarrolla de manera acelerada de cara a la globalización. Su crecimiento económico, urbano y demográfico crecen a ritmos acelerados. Monterrey es una de las ciudades con mayor índice de desarrollo humano (IDH) en México y entre las que más crecimiento económico y aportación al producto interno bruto (PIB) genera en el país. Sin embargo, la ciudad se enfrenta ante una serie de problemáticas urbanas, ambientales, sociales y culturales que necesitan atenderse. $\mathrm{Su}$ crecimiento urbano ha sido desmedido, deviniendo en un área metropolitana extensa, aunado a esto, la crisis ambiental que hoy en día enfrenta la ciudad la convierte en una de las más contaminadas de Latinoamérica. Por si no fuera poco, su centro urbano presenta problemas de abandono, degradación, pérdida de población y patrimonio. Éstas problemáticas son generalizadas en los centros de ciudades latinoamericanas, en donde el centro pierde jerarquía por la consolidación de las periferias (para ejemplos ver Rojas, 2004). A pesar del estado en el que se encuentra el centro, se parte de la idea de que tiene elementos culturales que pueden reforzar la identidad colectiva y revitalizarlo desde la perspectiva de la sostenibilidad cultural. El centro cuenta con equipamientos culturales, recursos patrimoniales y simbólicos que a partir de su reconocimiento y revalorización pudieran regenerar y convertir el centro en un ecosistema urbano resiliente y sostenible.

El objetivo de esta investigación consiste en analizar el centro de Monterrey a partir de la concentración de recursos culturales dentro de sus límites e identificar la existencia de clústers culturales donde pudieran estar sucediendo transformaciones urbanas alternativas a las oficiales. La intención es visibilizar y analizar desde una perspectiva distinta concentraciones de cultura dentro de un territorio y entender las dinámicas socio urbanas que los caracterizan. La aportación es analizar el centro desde un conjunto de valores culturales y que este método pudiera replicarse en contextos urbanos distintos. El análisis contribuye en el entendimiento de las dinámicas que suceden en el centro de Monterrey a través de diversas fuentes: documentación histórica, métodos cuantitativos y las valoraciones de la comunidad local sobre el territorio a modo de diagnóstico de cara a nuevas investigaciones o intervenciones sobre el sitio.

\section{Cultura, regeneración urbana $y$ sostenibilidad.}

Los aspectos culturales del desarrollo sostenible eran discutidos hasta hace poco desde el marco social. La inclusión de la cultura como cuarto pilar del desarrollo (Hawkes, 2001; Axelsson et al., 2013) ha hecho que se desarrolle el concepto de sostenibilidad cultural, ganado autonomía y visibilidad. Se define como el reconocimiento de 
la cultura en su entorno físico y social, identifica el valor de expresiones existentes en cada lugar a través de su patrimonio, geografía, historia, estilo de vida, arquitectura, tradiciones locales y costumbres, narrativas, fotografías, relatos y memoria colectiva (Soini y Birkeland, 2014; Duxbury et al., 2015).

El concepto de sostenibilidad cultural responde a valores propios del siglo XXI que además son de interés en la planeación urbana contemporánea como: el empoderamiento ciudadano, la cohesión social, la equidad, la identidad, la diversidad, el sentido de pertenencia y la resiliencia urbana (Oktay, 2011; Degen y García, 2012; Soini y Birkeland, 2014).

La cultura como herramienta de intervención urbana es un elemento esencial para el desarrollo de la ciudad porque combate la inequidad social (Degen y García, 2012) ayudando a mejorar aspectos socio económicos (Miles and Paddison, 2005; Degen y García, 2012) influyendo positivamente en la competitividad urbana. Por lo tanto, por su potencial para intervenir en la planeación urbana (Hawkes, 2001) es una alternativa viable para mejorar las condiciones y calidad de vida (Axelsson et al., 2013; Duxbury, 2014; Soini y Birkeland, 2014)

El marco de la sostenibilidad cultural permite a gobiernos locales identificar recursos culturales del entorno urbano para aplicar intervenciones de forma estratégica y alcanzar objetivos de desarrollo sostenible y comunitario. La cultura como elemento y estrategia de desarrollo debe integrarse a políticas públicas que reconozcan la ciudad como un ecosistema vivo y cambiante compuesto por una diversidad de recursos culturales. Antes de generar políticas al respecto, estos recursos deben ser reconocidos, cuestionados y valorizados por la comunidad (Bianchini y Parkinson, 1993; Bianchini y Ghilardi, 2007). Investigaciones demuestran que utilizar la cultura para promover la sostenibilidad genera impactos positivos tanto en la reestructuración del tejido urbano como en el desarrollo de comunidades locales (Lazarevic et al., 2015; Cuachi-Santoro, 2016). Por lo tanto, la sostenibilidad cultural no puede separarse de la parte social, por lo que propone un acercamiento integral y coalición entre políticas públicas, academia, sector privado y sociedad civil (Soini y Birkeland, 2014).

Las estrategias culturales que más se utilizan en la ciudad van desde proyectos de regeneración urbana basados en la creación de proyectos emblemáticos o equipamientos culturales diseñados por arquitectos de marca (Bianchini y Parkinson, 1993; Evans, 2005; García, 2004) como por ejemplo, el Guggenheim de Bilbao (Valdés Lange, 2018) o la red de parques-biblioteca en Medellín, Colombia (Duque Franco, 2015); hasta la creación de industrias creativas o culturales (Landry, 2003; Montgomery, 2003; Evans y Shaw, 2004), como el Poblenou en los 90 en Barcelona (Martí-Costa y Pradel i Miquel, 2011); o a partir de la creación de políticas culturales orientadas en apoyar y respaldar procesos de regeneración urbana existentes mediante la inclusión de agendas culturales que promueven eventos, exposiciones, conciertos o festivales (Bianchini y Parkinson, 1993; Landry y Bianchini, 1995; Duque Franco, 2015), como es el caso del proyecto Capital Europea de la Cultura (García, 2004).

Las estrategias culturales mencionadas no deberían ser replicables arbitrariamente, ya que los contextos urbanos, sociales y culturales de cada lugar son distintos entre sí teniendo recursos y valores propios que los conforman dándo un valor único a la comunidad. Como una manera de sistematizar el proceso de regeneración cultural a cualquier contexto, es importante, primero, reconocer los recursos culturales tangibles e intangibles del lugar. El valor de reocnocerlos, consiste en que la comunidad los reconozca como propios, fomentando regeneraciones urbanas integrales, deviniendo en más oportunidades de éxito. Una manera de identificar los recursos culturales de determinado lugar es mediante mapeo cultural. A continuación definiremos el concepto, su origen y las formas en que se aplica como herramienta de investigación y análisis.

\section{EI mapeo cultural como herramienta de análisis socio territorial.}

La cultura es parte importante de la agenda urbana del desarrollo (Dinardi, 2012), por lo que para proponer estrategias que busquen la sostenibilidad, es necesario valorizar los recursos culturales presentes en el territorio para la toma de decisiones multi-sectorial (Duxbury et al., 2015). La importancia de reconocer elementos culturales radica en que componen el ecosistema urbano, produciendo beneficios para sus habitantes como calidad de vida y desarrollo humano (Daniel et al., 2012). 
El mapeo cultural es una alternativa utilizada para cartografiar recursos culturales de un determinado territorio. Instrumento que permite la producción de conocimiento sobre un sitio o comunidad para reconocer las características culturales que los identifican. El mapeo cultural debe ser visto a modo de diagnóstico para proponer futuras estrategias de mejora y ayudar en la toma de decisiones (Freitas, 2015; Chiesi y Costa, 2015; Aliyah et al., 2017). Autores como Duxbury et al., (2015) definen el concepto como "modelo de investigación emergente", mientras que otros autores lo consideran más como una herramienta de análisis (Freitas, 2015). Si bien, el enfoque entre autores difiere, es importante manifestar la adaptabilidad del concepto, pudiendo aplicarse para el fin que sea requerido, ya sea como instrumento de análisis o como método de investigación. Por lo tanto, su aplicación y definición puede variar y adaptarse de acuerdo a la necesidad. Un elemento que no es negociable es la participación de la comunidad, jugando un rol fundamental a partir de sus valoraciones, reconocimientos y la puesta en valor de los recursos culturales locales (Duxbury et al., 2015).

Inicialmente, el concepto surge para investigar y proteger la diversidad cultural en pueblos indígenas (Crawhall, 2007; Duxbury et al., 205; Freitas, 2015). Sin embargo, se puede ampliar $\mathrm{su}$ uso hacia otros territorios y contextos, especialmente el urbano (Bianchini y Ghilardi, 2007). El objetivo del mapeo cultural consiste en identificar los recursos culturales de un lugar para tener una especie de compilación para a partir de ese reconocimiento, contribuir en la planeación urbana y en la implementación de acciones y estrategias orientadas hacia fomentar la sostenibilidad y la resiliencia socio urbana del lugar (Freitas, 2015; Duxbury et al., 2015; Aliyah et al., 2017). El mapeo cultural centra su enfoque tanto en el medio físico como en el social (Pillai, 2013). De ahí que sea indispensable reconocer las valoraciones de la comunidad local sobre el tejido urbano que habitan, ya que solo a partir de las apreciaciones locales es posible entender dinámicas existentes.

Losrecursosculturalessontangibleseintangibles. Los tangibles son cuantificables, espacios físicos como equipamientos culturales, organizaciones culturales, industrias culturales, patrimonio cultural, arquitectura $\mathrm{u}$ otros elementos materiales (Duxbury et al., 2015; Cuachi-Santoro, 2016; Jeannotte, 2016).
Los intangibles son cualitativos y se componen por valores y normas, creencias, narrativas, historias, memorias, relaciones, rituales, identidades $\mathrm{y}$ sentido de pertenencia (Duxbury et al., 2015:02; Longley y Duxbury, 2016; Cuachi-Santoro, 2016). Uno de los principales retos del mapeo cultural es cartografiar los recursos intangibles ya que al no ser cuantificables, se complica su identificación (Jeannotte, 2016). Sin embargo, su aportación es de gran valor ya que le dan sentido al tejido urbano por basarse en percepciones, valoraciones y narrativas de la comunidad local respecto al entorno donde habitan (Duxbury et al., 2015; Freitas, 2015; Cuachi-Santoro, 2016; Jeannotte 2016).

Siguiendo ciertos métodos y herramientas cualitativas es posible cartografiar recursos intangibles. Un ejemplo es partir del análisis de narrativas y memorias de actores clave y residentes locales (Chiesi y Costa, 2015; Jeannotte, 2016; Cuachi-Santoro, 2016), donde la finalidad es proveer una descripción sustancial de los elementos intangibles presentes. Las valoraciones locales fomentan el sentido de pertenencia (Chiesi y Costa, 2015) y la identidad local (Cuachi-Santoro, 2016). Por lo tanto, las valoraciones y percepciones de actores clave son una herramienta efectiva para construir mapas culturales que cuenten la historia de un lugar. Capturar narrativas es un método que se desarrolla de manera no lineal dotando de significado a un lugar, por lo que su validación y uso como material para mapear lo intangible debe seguir validándose como método de evaluación y reconocimiento del territorio.

Un mapeo cultural puede realizarse desde estrategias top-down y bottom-up (Freitas, 2015; Chiesi y Costa, 2015). La top-down se dirige por expertos, orientada a conseguir beneficios económicos basado en técnicas cuantitativas de análisis (Freitas, 2015; Chiesi y Costa, 2015). Mientras que la bottom-up es más colaborativa, basada en técnicas cualitativas en donde los residentes o actores locales definen el proceso y las estrategias a seguir a partir de necesidades locales (Freitas, 2015; Chiesi y Costa, 2015).

Dentro de los mayores retos del mapeo cultural está utilizar estrategias adecuadas para equilibrar la preservación de recursos culturales y por otro, implementar estrategias que promuevan el desarrollo sin comprometer la pérdida de identidad o vitalidad urbana (Hawkes, 2001; Freitas, 2015; CuachiSantoro, 2016). Además, el mapeo cultural ayuda a reconocer la existencia de zonas de concentraciones 
de cultura o clúster culturales. La identificación de un clúster cultural permite entender las dinámicas del territorio, influyendo de manera positiva en la planeación urbanística. Permite la creación de políticas públicas culturales y urbanas, asignación de fondos, creación o mejora de infraestructura, planeación pública urbana (Duxbury et al., 2015; Freitas, 2015), implementación de turismo cultural, rutas y paseos culturales, así como estrategias de regeneración urbana enfocadas en la recuperación de patrimonio cultural, histórico y arquitectónico (Cuachi-Santoro, 2016).

\section{Los clústers de sostenbilidad cultural.}

El mapeo cultural reconoce la localización y distribución de recursos culturales geográficamente. Ayuda a identificar zonas donde se concentran recursos culturales o se da una determinada actividad. Un clúster cultural es una zona que presenta concentración de equipamiento e industrias culturales, acompañado de políticas de desarrollo enfocadas a la generación y/o distribución de servicios culturales que mejoran la economía y competitividad de la ciudad (Zukin y Braslow, 2011; Zarlenga et al., 2016).

Los clúster culturales favorecen la revitalización de un área -abandonada o no- a partir del desarrollo económico y las actividades generadas por la cultura. Se producen desde diversas dinámicas sociales como a partir de la concentración espacial de industrias culturales, lo que genera beneficios económicos por su proximidad (Miles y Paddison, 2005; Zarlenga et al., 2013). Un ejemplo es el barrio de las industrias de la cultura (Cultural Industries Quarter) en Sheffield, Inglaterra el cual se compone por una concentración de más de 100 empresas destinadas a la cultura en una zona del centro de la ciudad (Moss, 2002). También estan aquellos producidos a partir de concentración de equipamientos culturales, los cuales usualmente se planean desde una iniciativa política que favorece la economía de escala (Miles y Paddison, 2005; Zarlenga et al., 2016). Como ejemplo podemos mencionar la Isla de los Museos en Berlín, en donde en un área determinada se concentran 5 museos. Otros clúster culturales se producen bajo una estrategia de regeneración que involucra la creación de distritos culturales para potenciar zonas de la ciudad, creando espacios mixtos de difusión y consumo cultural (Zarlenga et al., 2016). Un ejemplo de esta tipología es el Temple Bar en Dublín, barrio donde se concentra gran cantidad de equipamientos culturales y artísticos, y que a su vez también es sede de mercados, industrias y eventos culturales. Su modelo de consolidación y gestión se diferencía del resto porque surge desde la iniciativa pública mientras que después es la iniciativa privada junto con la sociedad civil quienes genera dinámicas de acción e intervención desde el ámbito cultural, creativo y/o de ocio (Zarlenga et al., 2016).

Los tres modelos se producen a partir de iniciativas top-down por parte del sector público o asociaciones entre público-privados (Bianchini y Parkinson, 1993; Miles y Paddison, 2005). También existe un modelo alternativo: clúster culturales producidos a partir de dinámicas de barrio, desde enfoques bottom-up, sin planeación política o administrativa que los sustente y que han logrado regenerar tanto el tejido social como urbano (Zarlenga et al., 2016). Surgen desde dinámicas bottom-up, en donde los residentes junto con creadores y consumidores de diversas formas de cultura generan una concentración urbana de espacios y movimientos culturales produciendo efectos positivos (Landry, 2003). Uno de los casos más representativos es el de Villa Crespo, barrio argentino que se transforma a partir de la gran cantidad de artistas que se asientan allí, favoreciendo la economía y recuperación del lugar.

Los beneficios producidos por los clústerculturales van desde ámbitos estéticos, urbanos, económicos e inclusive sociales, debido a la densidad de relaciones e intercambios que suceden entre agentes (Markusen, 2007; Wu, 2005; Zarlenga et al., 2016). Los procesos bajo los cuales se producen los clusters culturales permite diferenciar las características y dinámicas de consolidación y funcionamiento, permitiendo reconocer el territorio de manera más integral a partir de estrategias específicas de intervención, actuación y conservación.

\section{Metodología.}

Para identificar la existencia de clúster culturales se utiliza una combinación de métodos cuantitativos, cualitativos y cartográficos. Se parte del análisis urbanístico junto con su historia y evolución, así como del planeamiento y las políticas implementadas anteriormente (Garza-Rodríguez, 2015). Se hace 
una caracterización socio demográfica a escala de Área Geo Estadística Básica (AGEB), -unidades territoriales utilizadas por el Instituto Nacional de Estadística y Geografía (INEGI)-, junto con un levantamiento cartográfico de los recursos culturales tangibles, realizado a partir de búsqueda en bases de datos del INEGI. Una vez obtenidos los datos, se categorizan las variables culturales en: (1) Espacio Público, (2) Espacios Culturales Independientes (ECI), (3) Equipamientos Culturales, (4) Centros Educativosy (5)Elementos Patrimoniales-Simbólicos (ver tabla 2); se aplica un ratio de 600 metros a cada categoría por ser la distancia recomendada que favorece la proximidad (Rueda et al., 2013).

Cuadro 1. Categorización propuesta para cartografiar Recursos Culturales Tangibles.

\begin{tabular}{|l|l|}
\hline \multicolumn{1}{|c|}{ Categorías } & \multicolumn{1}{c|}{ Recursos Culturales Tangibles } \\
\hline $\begin{array}{l}\text { Equipamientos } \\
\text { Culturales }\end{array}$ & $\begin{array}{l}\text { Museos, Teatros, Bibliotecas, } \\
\text { Recintos para eventos }\end{array}$ \\
\hline Centros Educativos & $\begin{array}{l}\text { Escuelas secundarias, preparatorias y } \\
\text { universidades }\end{array}$ \\
\hline $\begin{array}{l}\text { Elementos } \\
\text { Patrimoniales- } \\
\text { Simbólicos }\end{array}$ & $\begin{array}{l}\text { Centros espirituales/religiosos y } \\
\text { Monumentos históricos }\end{array}$ \\
\hline Espacio Público & Plazas públicas y áreas verdes \\
\hline $\begin{array}{l}\text { Espacios Culturales } \\
\text { Independientes } \\
\text { (ECI) }\end{array}$ & $\begin{array}{l}\text { Espacios culturales, galerías de arte, } \\
\text { editoriales, librerías, café cultural, } \\
\text { comercio local, antigüedades }\end{array}$ \\
\hline \multicolumn{2}{|c|}{ Fuente: Elaboración propia. } \\
\hline
\end{tabular}

El ratio permite generar un mapa de calor de Intensidad Cultural. En él, se visualizan zonas del territorio con mayor densidad o concentración de recursos culturales. Obtenido el mapa de calor se cruza en formato vectorial información socio demográfica a nivel de AGEB -la única disponiblepara analizar el perfil socio-demográfico.

Se distinguen 3 niveles de intensidad en el mapa de calor: alto, medio y bajo. Las intensidades hacen referencia a la proximidad de recursos culturales. Bajo significa poca proximidad entre ellos, mientras alto significa alta proximidad. Por lo tanto, se identifica el clúster cultural a partir de la alta proximidad de sus recursos. Una vez identificado, se sigue con la valoración de recursos culturales intangibles mediante la aplicación de entrevistas en profundidad a informantes clave que habitan dentro del clúster. El perfil de los informantes está compuesto por académicos, representantes vecinales, de colectivos y de espacios culturales independientes. Se realizan
15 entrevistas en total, en donde el objetivo es emplear una muestra representativa a través de un análisis en profundidad y no en extensión. Los informantes se elijen por su interés por mejorar el centro. Se busca que tengan distintos perfiles para obtener respuestas diversificadas. La entrevista está compuesta por 14 preguntas semi-estructuradas, donde se pregunta sobre los espacios urbanos que conforman el clúster, sobre la existencia y valoración de espacios públicos y áreas verdes, sobre cuáles son los más utilizados y por quién, sobre el tipo de población que los frecuenta. Se pregunta también sobre características arquitectónicas del clúster: si existen edificaciones representativas o identificativas y cuales tienen mayor valor para la comunidad. También se explora si el clúster cuenta con grupos u organizaciones culturales, y de ser así, identificarlos y responder cuales son los más activos. La entrevista termina preguntando sobre los cambios urbanos sucedidos en la zona, sobre quien los ha promovido, si ha mejorado o empeorado y con propuestas de mejora para el barrio. Consideramos las entrevistas como una especie de cartografía verbal que nos permite dibujar y entender desde un sentido narrativo el centro de Monterrey y el clúster cultural. 


\section{Resultados.}

A continuación se muestran los resultados obtenidos de acuerdo a la distribución espacial de los recursos culturales tangibles, mostrando su localización en el territorio y la proximidad entre unos y otros. Además se discuten y analizan las valoraciones de actores clave sobre el clúster identificado, con la finalidad de que en conjunto, tanto el análisis de la distribución espacial como las valoraciones, permitan generar una diagnosis sobre las dinámicas generadas dentro del clúster cultural.

Identificación de clúster culturales en el centro de Monterrey mediante mapa de Intensidad Cultural.

El centro de Monterrey es un territorio que cuenta con un fuerte tejido cultural desde el enfoque de proximidad, ya que casi todo el polígono está cubierto por una tipología específica de recursos culturales (ver fig. 1). De estos recursos, en el centro predominan los Elementos SimbólicosPatrimoniales los cuales se conforman por Monumentos Históricos, Centros Espirituales y Religiosos y Patrimonio Histórico-Arquitectónico. Los Centros Educativos son los recursos culturales que después de los Elementos SimbólicosPatrimoniales predominan; éstos se componen en su mayoría, por universidades, preparatorias y escuelas secundarias. Resulta irónico que los Equipamientos Culturales son los que menor presencia tienen en el centro de Monterrey, los cuales se pensaba podrían predominar. En cuanto al Espacio Público, compuesto por plazas y áreas verdes, si bien son escasas, su principal ventaja es que al menos dos de ellos son grandes espacios públicos como es el cerro del Obispado, la Macroplaza y el Parque Fundidora, aunque aún así, son insuficientes. Investigaciones señalan que una ciudad con áreas verdes de calidad produce orgullo ciudadano porque genera entornos saludables tanto para el capital social como para el natural (Oktay, 2011).

Figura 1. Mapa del total de recursos culturales cartografiados en el centro de Monterrey.

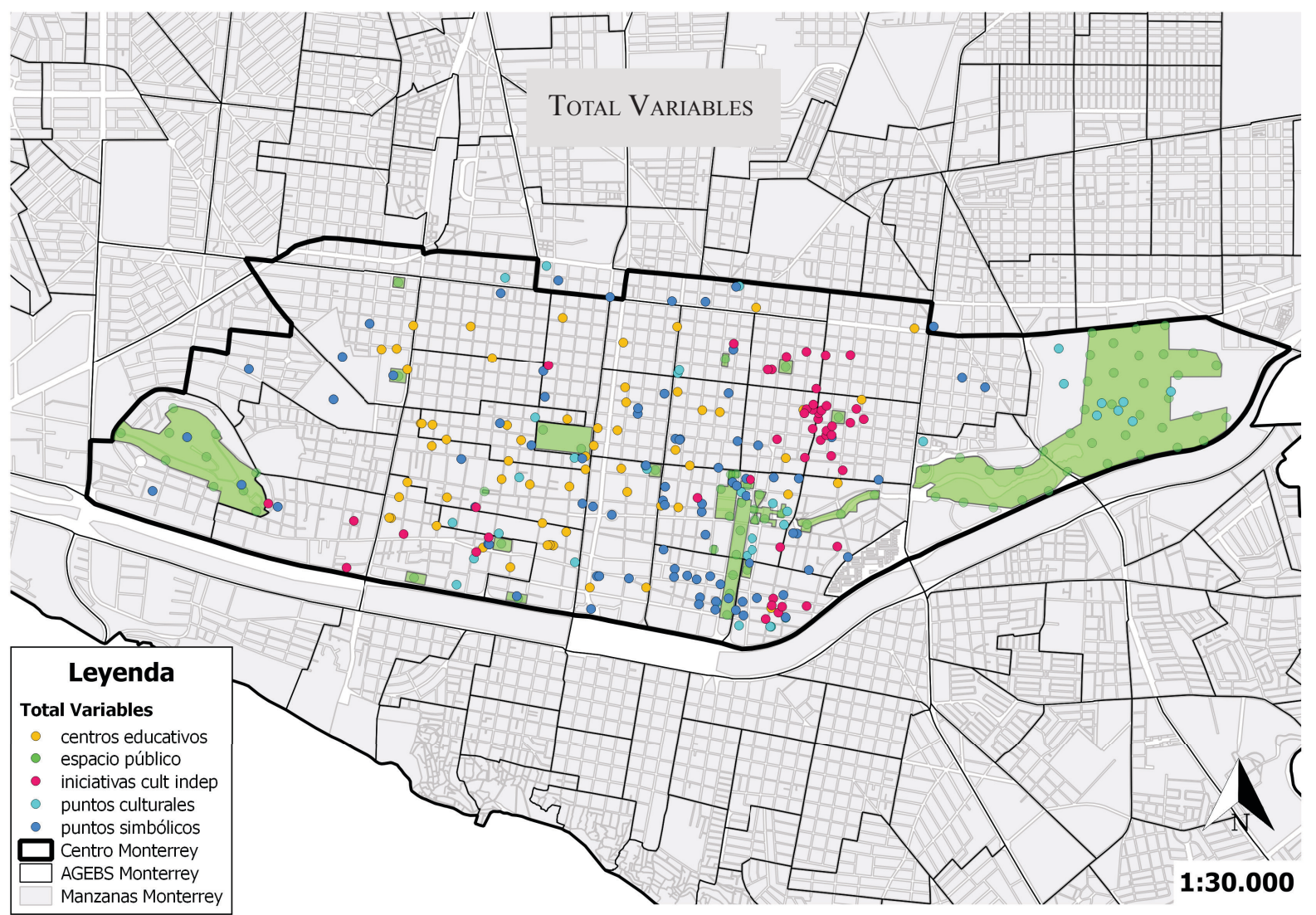

Fuente: Elaborado por Helena Grau y la autora. 
Figura 2. Mapa de calor de intensidad cultural en el centro de Monterrey.

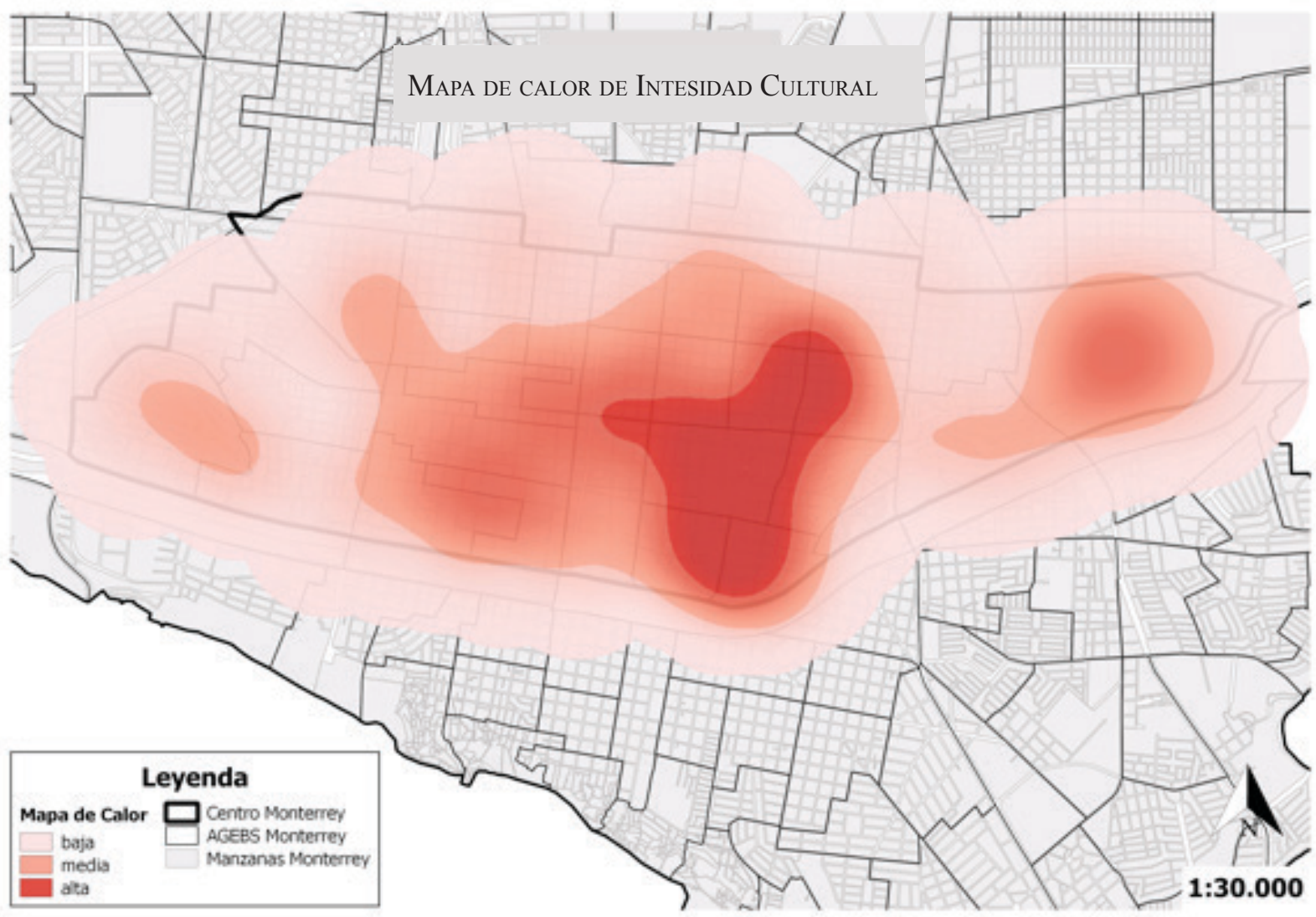

Fuente: Elaborado por Helena Grau y la autora.

Los cálculos arrojan resultados similares para cada nivel de intensidad (ver tabla 1), por lo que no representan diferencias significativas entre zonas. Es importante aclarar que estos cálculos se obtienen del Censo de Población disponible más reciente el cual data del 2012, antes de que se produjeran algunas de las transformaciones urbanísticas ligadas a la gestión y promoción cultural del centro. Los resultados muestran, aparentemente, que las características de la población habitante en el centro a inicio de la década eran bastante homogéneas, por lo que es necesario contrastarlo desde un enfoque cualitativo para valorar si se han producido cambios en estos términos.
El mapa de Intensidad Cultural permite reconocer el centro a partir de la distribución y proximidad de los recursos culturales existentes y conocer características socio demográficas y económicas de la población que habita en cada intensidad, como por ejemplo la edad de la población, la Población Económicamente Activa (PEA), Ocupados, Desocupados, población de 18 años y más con educación pos-básica, es decir, educación más allá del nivel secundaria y las viviendas particulares habitadas. A partir de estas variables es posible entender el territorio desde características socio demográficas y económicas.

Cuadro 2. Resultados en porcentaje del perfil socio-demográfico por intensidades (valores relativos a la población de cada grado de intensidad).

\begin{tabular}{|c|c|c|c|c|c|c|c|c|c|}
\hline & Pob Inf \% & Pob Joven \% & $\begin{array}{c}\text { Pob Adulta } \\
\%\end{array}$ & $\begin{array}{c}\text { Pob } 65 \text { y } \\
\text { más \% }\end{array}$ & PEA \% & P Ocup \% & P Desocup \% & $\begin{array}{c}\text { P18y > con } \\
\text { Edu Pos } \\
\text { Básica \% }\end{array}$ & $\begin{array}{c}\text { Viviendas } \\
\text { Particulares } \\
\text { Hab \% }\end{array}$ \\
\hline $\begin{array}{r}\text { DU BAdA } \\
\text { RT IEDIA } \\
\text { WTALTA }\end{array}$ & $\begin{array}{l}13.92 \\
12.60 \\
12.52\end{array}$ & $\begin{array}{l}14.99 \\
15.42 \\
13.53\end{array}$ & $\begin{array}{l}51.89 \\
5.37 \\
5.17\end{array}$ & $\begin{array}{l}16.90 \\
16.67 \\
17.55\end{array}$ & $\begin{array}{l}45.01 \\
47.55 \\
49.15\end{array}$ & $\begin{array}{l}96.18 \\
96.13 \\
\mathbf{9 6 . 0 4}\end{array}$ & $\begin{array}{l}3.78 \\
3.83 \\
4.95\end{array}$ & $\begin{array}{l}\mathbf{5 3 . 6 3} \\
\mathbf{5 4 . 3 1} \\
\mathbf{5 6 . 3 3}\end{array}$ & $\begin{array}{l}70.48 \\
67.58 \\
55.71\end{array}$ \\
\hline $\begin{array}{l}\text { IEDIA } \\
\text { GENTRO }\end{array}$ & 1220 & 14.51 & 54.25 & 16.31 & 46.66 & 96.30 & 3.51 & 51.01 & 64.33 \\
\hline
\end{tabular}


Sin embargo, este análisis cuantitativo no demuestra el impacto que ha tenido la cultura en el entorno urbano, es decir si se han producido fenómenos urbanos diferenciados, desde procesos top-down o bottom-up y que además, ayuden a profundizar si ha habido efectos sobre su población. Es por ello que recurrimos al análisis cualitativo partiendo de revisión documental, histórica y de la planificación urbana y, muy especialmente a partir de las entrevistas realizadas a actores clave con la finalidad de responder a este gap.

\section{Construyendo una narrativa: Valoraciones locales del clúster cultural.}

El clúster cultural identificado representa un área territorial de 150 hectáreas. El mapa (Fig.2) identifica la concentración de recursos culturales en el centro de Monterrey. Se contabilizan 96 recursos culturales, de los cuales 31 son elementos simbólico-patrimoniales, 31 son Espacios Culturales Independientes, 20 son Centros Educativos y 14 son Equipamientos Culturales. En cuanto al espacio público, éste suma un total de 14 hectáreas entre plazas públicas y áreas verdes, representando el $21 \%$ del territorio del clúster. La población que habita en la intensidad alta es del $9 \%$, valor relativo a la población total del centro. En valores relativos al clúster, la estructura poblacional se conforma mayormente por población adulta (43\%), mientras que el $18 \%$ es población mayor a 65 años. La población infantil y joven se mantienen constantes (entre el 13\% y 14\%). El 50\% de los habitantes son económicamente activos, mientras que el $56 \%$ cuenta con estudios más allá de la preparatoria (bachiller), lo que refleja que la población que habita en el centro cuenta con un nivel educativo alto. De $68 \%$ de viviendas totales, solo $38 \%$ están habitadas dentro del clúster, lo que indica que un $30 \%$ de las viviendas, poco menos de la mitad, están en situación de abandono. En resumen, los resultados muestran que el centro de Monterrey cuenta con un clúster cultural compuesto por una diversidad de recursos culturales tangibles, mientras que la estructura poblacional que habita próximo a estos recursos es mayormente adulta, seguida de población envejecida, población que además cuenta con alto nivel de estudios.

Partiendo del reconocimiento de estas características, pasamos a analizar las valoraciones obtenidas a través de las entrevistas. Analizaremos a partir de narrativas o story-telling (Jeannotte, 2016), los resultados obtenidos con la finalidad de crear una discusión analítica sobre lo que perciben los actores clave sobre los recursos culturales del centro.

Los entrevistados reconocen el centro de Monterrey como un tejido urbano que contiene elementos culturales de gran valor por ejemplo, a partir de sus equipamientos culturales o zonas de interés cultural como monumentos históricos. El interés por "devolverle la vida al centro" es un discurso que ha estado presente constantemente en la administración municipal, ya que reconocen que desde hace varias décadas el centro se ha convertido en un lugar deteriorado, lo que ha devenido en despoblamiento y a su vez, en mayor inseguridad y abandono. Esto es algo que tienen claro los entrevistados, por una parte reconocen que el centro cuenta con muy poca población habitante, pero por el otro, argumentan que en el día es un lugar dinámico donde se dan actividades relacionadas principalmente al comercio y servicios. Argumentan que el abandono se presenta en las noches, cuando el centro se vacía de la población que trabaja ahí. Se reconocen los esfuerzos que tanto la administración estatal como municipal han realizado por regenerar y revitalizar el centro, materializándose a partir de propuestas de regeneración urbana para dinamizar la zona. Por ejemplo, la primer regeneración urbana que tiene lugar en el centro es la que se da para construir la Macroplaza a inicios de la década de los 80. Después de este proyecto de regeneración que implicó la destrucción de manzanas que contenían edificaciones de carácter histórico-patrimonial, siguieron otros proyectos de regeneración de gran escala como la construcción del Museo de Historia Mexicana junto con el pequeño canal Santa Lucía que años después recuperaría de manera artificial el "ojo de agua" creando un gran espacio público que conecta la Macroplaza con el Parque Fundidora.

Las estrategias bajo las cuales se producen las regeneraciones urbanas en el centro se han producido desde modelos top-down, a partir de proyectos creados, diseñados, gestionados y financiados por el sector público. Sin embargo, estas regeneraciones que involucran gran inversión y que además, tienden hacia la monumentalidad, no han logrado los objetivos esperados. Prueba de ello es el abandono generalizado que aún prevalece y que sigue extendiéndose. Si bien, se han creado nuevos equipamientos junto con mejoras en la calidad del espacio urbano, esto ha sido a costa de la pérdida y destrucción de patrimonio histórico y arquitectónico construido y el desplazamiento de la población, como lo ilustra el siguiente argumento: 
"De entrada aqui hubo un proceso de urbanización autoritario para construir lo que es la Macroplaza, hubo una expulsión de muchísimas familias, la destrucción de muchas casas, hogares, esa expulsión hizo que crecieran otras zonas de la ciudad..." (Representante de colectivo).

La migración de población se pude ver influenciada por diversos factores, en algunos casos por la pérdida de identidad colectiva la cual se relaciona directamente con la pérdida del sentido de pertenencia entre residentes locales. En ocasiones, el desplazamiento responde a fenómenos orientados al encarecimiento de la zona, al término conocido como gentrificación. El sentido de pertenencia refleja las experiencias en un lugar, pero también los significados culturales, religiosos, históricos y personales vividos en el sitio, esto es lo que moldea interacciones sociales pasadas y actuales (Chapin y Knapp, 2015), de ahí la importancia de mantenerlo vigente.

Por otro lado, reconocer y preservar el patrimonio cultural permite entender la historia del lugar, siendo un punto de partida de cara hacia regeneraciones y políticas urbanas que consideren el perfil cultural (Cuachi-Santoro, 2016). Por lo que la conservación del patrimonio cultural, histórico y arquitectónico podría ser un elemento que refuerce el sentido de pertenencia de la comunidad.

Por ejemplo, en el clúster identificado, un $30 \%$ de las viviendas están abandonadas. El abandono no sólo es visible en viviendas, sino también en espacios públicos, locales comerciales, construcciones sin terminar, así como calles enteras. La principal causa que produce esta situación es que no existe un programa de fomento público que promueva la rehabilitación y/o ocupación de estos lugares o algún tipo de subsidio que permita el acceso a vivienda en el centro. Acciones como éstas, han logrado rehabilitar y repoblar centros de ciudad en otros contextos, como por ejemplo, en Santiago de Chile (Contreras Gatica, 2011). El enfoque que el sector público ha puesto en marcha para regenerar el centro es a través de de planes y programas de regeneración urbana que no terminan por consolidarse debido a factores como falta de continuidad en administraciones o falta de presupuesto. Por su parte, el sector privado impulsa regeneraciones a partir de la construcción de edificios de usos mixtos, usualmente construcciones verticales de gran altura con el que buscan 'redensificar' el centro y resolver el tema de vivienda. Sin embargo, aún está por verse que tanto resuelven estas estrategias ya que el público al que se dirigen tiende a ser de un estrato medio - medio alto y las opciones de vivienda no son muy atractivas.

"El abandono es importante, hemos dejado de pensar en el centro como un lugar habitable, hay muchas casas abandonadas y no hay un seguimiento, ni un programa para reactivarlo" (Representante de colectivo).

La zona en donde se ubica el clúster cultural se caracteriza por ofrecer dinámicas económicas, sociales y culturales heterogéneas. El uso de suelo está destinado a bares, discotecas, antros, turismo y a la escena cultural (museos, galerías de arte) de Monterrey y en menor medida, con uso de suelo habitacional. Destaca por ejemplo, el caso del polígono del Barrio Antiguo. Conocido como el centro histórico de la ciudad, desde hace más de 20 años se ha convertido en el polo de ocio nocturno del centro y del área metropolitana. Esto ha originado que el sector público produzca actuaciones urbanísticas enfocadas en mejorar su imagen urbana a través de intervenciones estéticas (Prieto González, 2011). El polígono también se caracteriza por ser sede de la escena cultural y bohemia de la ciudad, con la presencia de artistas que tienen sus talleres de arte en el barrio junto con comercios destinados a la venta de antigüedades, creación de mobiliario o espacios que promueven el arte y la cultura. Una de las desventajas de la actividad nocturna del Barrio Antiguo es que ha provocado malestar y descontento entre residentes locales, generando desplazamiento de población y pérdida de identidad. Los establecimientos nocturnos además de alterar el contexto urbano y poblacional alteran la tipología arquitectónica al modificar construcciones declaradas patrimonio arquitectónico. A pesar de estar catalogadas por instituciones públicas de protección del patrimonio como el Instituto Nacional de Antropología e Historia (INAH), no son protegidas para evitar su modificación y/o destrucción. Con el paso de los años estos sucesos generan pérdida de identidad y desarraigo del lugar por no conservar la arquitectura original, ni promover dinámicas que reflejen su pasado histórico y que mantengan presente la memoria colectiva. 
"Barrio Antiguo es la zona de antros y galerías de arte, se ha tratado de decir que ahí hay una comunidad pero en realidad es una comunidad de negocios, no de habitantes" (Representante de colectivo).

Por otra parte, dentro del clúster se encuentra el Barrio de La Luz, polígono orientado a las artes y oficios del Monterrey antiguo. El barrio era lugar de talleres y espacios destinados a ofrecer servicios como sastrerías, carpinterías, joyerías, talleres de arte, ferreterías, zapaterías. Hoy en día, aún permanecen algunos de estos establecimientos, reforzando esta identidad. Es el caso, por ejemplo de una ferretería que tiene más de 30 años en el barrio, de un taller relojero o del taller de piñatas del barrio. La permanencia y conservación de los pequeños comercios es un fenómeno que se reconoce como parte del barrio, dándole un valor añadido a su tejido socio urbano por reforzar su identidad como área destinada a ofrecer servicios culturales específicos. Algo similar sucede en el barrio de Savamala en Serbia, en donde iniciativas culturales independientes han logrado impulsar y revitalizar culturalmente esta área a partir de enfoques bottom-up (Lazarevič et al., 2015).

Las entrevistas permiten saber que el Barrio de la Luz ha sido uno de los pocos que no ha perdido población. A inicios del 2013, en el polígono se ha dado un fenómeno interesante de aparición de espacios culturales independientes (ECI) destinados a talleres de arte, galerías, librerías, escuelas artísticas y cafés culturales que surgen desde la misma sociedad civil, apareciendo de manera autónoma para llenar el gap cultural que la actuación pública ha dejado en la ciudad. De paso ayuda a promover la descentralización de la cultura. El análisis de recursos culturales previamente realizado arroja la existencia de una marcada concentración de espacios culturales en el barrio, contabilizándose alrededor de 20 . Esta proliferación de espacios culturales ha desencadenado efectos positivos tanto en la población como en el tejido urbano del lugar ya que al estar agrupados en un determinado lugar, las redes colaborativas entre artistas, dueños, habitantes y ocupantes de los espacios han reforzado la identidad colectiva del barrio. Las redes sociales contribuyen en mantener la vitalidad del lugar ya que se utilizan para publicar eventos, talleres, conferencias o festivales, permitiendo mayor difusión. La escala de barrio permite que haya un reconocimiento entre los habitantes y ocupantes del barrio, generando confianza entre vecinos.

"Toda la gente se conoce, esta lleno de lugares como comercios pero culturales porque tienen una gran parte de cultura por ejemplo la ferretería, la papelería, o sea cosas que ya en otros lugares no existen" (Activista del clúster).

Por otro lado, los edificios arquitectónicos que más valor tienen para la comunidad varían de un lugar a otro dentro del clúster. Al contener un espacio territorial tan amplio, se visibiliza la existencia de distintas realidades arquitectónicas dentro de su tejido. Por ejemplo, en la parte sur del clúster, las edificaciones que más valor tienen para la comunidad local son los equipamientos culturales y religiosos. Esto se debe a que en la zona predominan estos equipamientos, los cuales tienden hacia la monumentalidad y a lo simbólico. Entre los más representativos destacan el Palacio de Gobierno Estatal, la Capilla de los Dulces Nombres y el Museo de Arte Contemporáneo (MARCO), por mencionar algunos. Todos ellos corresponden a épocas distintas de construcción y a movimientos arquitectónicos muy distintos entre sí.

"La Capilla de los Dulces Nombres tiene un valor histórico [...] el Palacio de Gobierno Estatal es muy importante, es un símbolo de la ciudad, del poder, pero también es símbolo turístico, de identidad, también es el lugar por excelencia de las protestas, de meetings políticos, huelgas de hambre" (Investigador).

Por otro lado, en la parte norte del clúster, las edificaciones que más valor tienen para la comunidad son la iglesia de la Luz y la plaza. Esto se considera lógico ya que es de los pocos barrios -sino es que el único- que dentro de su forma urbana cuenta con plaza e iglesia propia. La plaza es un lugar de recreación y ocio para los habitantes, siendo el punto de reunión vecinal. También destacan casonas antiguas de estilo neocolonial, las cuales no están catalogadas como elementos patrimoniales pero representan un fuerte valor para la comunidad ya que son ejemplo tangible del pasado histórico del barrio. Las entrevistas identifican que se cuenta con un alto grado de reconocimiento del paisaje urbano por parte de los entrevistados. Sin embargo, el problema al que 
se enfrentan la mayoría de los inmuebles es que no están protegidos por instituciones del sector público, por no aparecer dentro de un catálogo de protección. En la zona norte del clúster no se han dado procesos de depredación de patrimonio o de arquitectura tan graves e invasivos como los que se han dado en otras partes del clúster, en donde ha habido procesos de destrucción del patrimonio de manera más generalizada. El derrumbe de los inmuebles, sepulta la identidad y memoria arquitectónica del lugar, la cual se sustituye por nuevas edificaciones que contrastan con la tipología de escala del centro, convirtiéndose en el peor de los casos, en áreas de estacionamiento.

La pérdida del patrimonio construido no es un fenómeno aislado del centro de Monterrey. Sucede en todo el mundo, desde Dublín, con la inminente destrucción del edificio histórico y protegido Boland's Mill, como parte de un plan de regeneración urbana para modernizar la zona, hasta la ciudad de Buenos Aires, en donde una gran cantidad de patrimonio arquitectónico se ha perdido para dar lugar a nuevas edificaciones o plazas de estacionamiento público. La importancia de revalorizar y conservar edificaciones patrimoniales -o de valor para la comunidad- radica en que contribuyen en la consolidación de la identidad colectiva de los habitantes y en su sentido de pertenencia (Cuachi-Santoro, 2016). Proteger y revalorizar el patrimonio es uno de los grandes retos para alcanzar la sostenibilidad cultural en la ciudad, ya que una de sus premisas clave es poner en valor las características locales del lugar.

Una forma en la que se manifiesta un intento de recuperación y rehabilitación del espacio urbano del centro se produce a partir de la realización de actividades culturales. Por ejemplo, está el que se da los domingos en el Barrio Antiguo con el 'Callejón del Arte' o 'Callejón Cultural', en donde una calle que atraviesa el barrio es cerrada a la vialidad (9am$8 \mathrm{pm})$ para convertirse en un bazar cultural que ofrece artesanías, antigüedades y obras de artistas locales. La venta se complementa con la presencia de escenas artísticas como conciertos o representaciones de arte escénico en la calle. El Callejón del Arte surge a inicios del 2004 como un programa público para promover la cultura en la ciudad, el cual se ha mantenido a lo largo de 18 años. Como en todo, hay opiniones a favor y en contra del proyecto. Residentes locales están en descontento por la ocupación invasiva alrededor de sus viviendas. También hay quien dice que las intenciones en un inicio eran buenas y positivas y que hoy en día, el Callejón del Arte se ha convertido en un tianguis. Sin embargo, lo destacable en una ciudad en donde hay poco espacio público y se hace poca vida en la calle es la dinámica generada alrededor de esta actividad, que se mantiene a lo largo de los años y que además, permite el recorrido peatonal de una calle que normalmente solo es transitable en coche. Con el paso de los años, ha ido en aumento la ocupación de inmuebles en los alrededores del callejón como restaurantes, librerías, y galerías de arte. Produciendo una mayor atracción para los habitantes de la ciudad creando mayor espacios de recreación y ocio que promuevan la vida en la calle. Otras manifestaciones culturales alternas que se dan en el clúster son a partir de la generación de eventos, festivales, conciertos al aire libre y expresiones de arte urbano.

"Ahora hay mucho arte urbano en varias paredes de calles de toda esta zona, arte urbano que ha sido de artistas urbanos individuales o por ejemplo del festival Callegenera, este festival el año pasado fue puro centro de la ciudad, todo lo hicieron en el centro y los murales están increibles"(Lider de colectivo).

La mayor cantidad de manifestaciones artísticas y culturales que tienen lugar en el centro ocurren dentro del clúster, esto se explica debido a la alta concentración de artistas y espacios de carácter culturalubicados allí. Un ejemplo de manifestaciones artísticas son las expresiones de arte urbano realizadas en propiedades privadas con permiso de los dueños. Se realizan murales urbanos con la finalidad de transformar de manera visual la estética del barrio, argumentando que es preferible ver una obra de arte urbano en un edificio que verlo en estado de abandono, degradado y sin mantenimiento; de esta forma, los murales se convierten en una forma de acercar el arte a los ciudadanos además de ser un indicador de resistencia social ante el abandono de los inmuebles.

"La barda de enfrente estaba bien fea $y$ buscamos quien se la quería aventar y 'El Doce' (artista visual local) se la aventó y mejoró mucho con el grafiti y los arboles; ya no se ve abandonado" (Dueña ECI). 
En ciudades mexicanas como en Xanenetla, en Puebla, el muralismo urbano ha sido utilizado como estrategia de regeneración urbana, realizada desde un enfoque bottom-up. A través de él, se fortalece la identidad colectiva del barrio y la cohesión social, produciendo una revalorización cultural del lugar y actuando a modo de resistencia hacia procesos de gentrificación (Kurjenoja e Ismael, 2015). Por lo tanto, el muralismo y el arte, son alternativas para mejorar la imagen urbana del lugar produciendo efectos positivos en los residentes como apropiación del espacio, sentido de pertenencia y valorización. Ejemplos como este, también suceden en el centro de Monterrey, como el caso del barrio del Nejayote, barrio degradado y abandonado que ha transformado su imagen urbana a través de la mejora estética de sus aceras, creando imágenes relativas a la identidad local de la ciudad mediante la técnica del trencadís, esto es, con pedacería de mosaicos de colores.

Por último, se ha visto la llegada de nuevos vecinos, sobre todo en la parte norte del clúster, jóvenes artistas en busca de un lugar con dinámicas de barrio, -tan difíciles de encontrar hoy en día en la ciudad-, en donde es posible hacer vida de manera compacta. Esta cualidad urbana atrae a jóvenes artistas a ubicar sus galerías, talleres de arte o espacios culturales en esta zona, por ser uno de los pocos lugares en el centro - si no es que el único- en donde se puede vivir de manera más cohesionada y alternativa a lo que conocemos en la ciudad.

Los resultados obtenidos reconocen que dentro del clúster cultural existen distintas zonas compuestas por características específicas muy diferenciadas como uso de suelo, perfil de población, elementos arquitectónicos, dinámicas culturales que no pueden valorizarse desde una visión generalizada. Por lo tanto, el clúster cultural se diferencia en dos nodos que responden y provocan distintas valoraciones y percepciones, los cuales se analizarán de manera independiente en futuras publicaciones. Esto con la finalidad de reconocer qué estrategias tanto urbanas como sociales y culturales se desarrollan en cada nodo para entender dinámicas y procesos de regeneración urbana alternos.

\section{Conclusión.}

Este trabajo contribuye al estudio de la sostenibilidad cultural en el urbanismo y en las formas en que la cultura se manifiesta en la geografía urbana, específicamente el trabajo contribuye en el uso y entendimiento de la técnica de mapeo cultural como herramienta de diagnosis e instrumento de análisis socio urbano en un contexto latinoamericano. La investigación explora a través de la revisión de literatura científica las formas en que la cultura se materializa como estrategia y herramienta de regeneración urbana a través de proyectos $\mathrm{y} / \mathrm{o}$ actividades culturales específicas en el territorio urbano. En los últimos años se ha reconocido la importancia de la cultura como elemento y motor de desarrollo urbano (Degen y García, 2012; Jeannotte, 2016), por lo que su uso como respuesta a problemáticas socio urbanas cada vez es más utilizado por el abanico de intervenciones que puede generar (Zukin, 1995; Miles and Paddison, 2005). De esta forma, la cultura se torna el mecanismo de transformación, ejerciendo un rol integrador entre esferas que actúa como conciliador entre instituciones y actores (Lazarević et al., 2015).

En el caso del centro de Monterrey, el mapeo cultural ayuda a conocer la distribución geográfica de los recursos culturales del territorio, identificando un clúster reconocido a partir de la concentración de recursos culturales. Se identifica una zona en donde existe un mayor grado de concentración o densidad de recursos culturales en el territorio, de esta forma se reconocen zonas en donde se da una mayor actividad cultural en el centro, a partir de los recursos culturales tangibles ahí presentes. En el clúster cultural identificado existen realidades y procesos de regeneración y consolidación distintos. Por una parte, está la zona que corresponde a un modelo orientado a la concentración de toda clase de equipamientos desde culturales y educativos hasta de consumo y ocio nocturno (MacroplazaBarrio Antiguo), mientras que otra zona está produciendo dinámicas culturales a partir de aglomeración de artistas y espacios culturales que trabajan de manera colaborativa por mejorar el barrio, influyendo de manera positiva en mejorar la cohesión social de la zona (Barrio La Luz).

El presente estudio debe verse como un modelo estratégico para futuras investigaciones y propuestas de intervención urbanística desde la perspectiva cultural. Donde se consideren tanto equipamientos culturales como actividades e implementación de políticas públicas urbanas para mejorar el estado actual del centro desde un enfoque 
cultural. Finalmente, se reconoce que hacen falta más contribuciones de sentido práctico hacia la búsqueda de la relación entre regeneración urbana y sostenibilidad cultural, así como manifestaciones en el entorno socio urbano, sobre todo en el ámbito latinoamericano. Este estudio pretende ser una primera aproximación, sin embargo se reconoce la necesidad de considerar más factores que ayuden a tejer la realidad cultural de un territorio desde los agentes que la provocan, a partir de sus dinámicas generadas e intervenciones realizadas. $\mathbf{C}$

\section{Referencias bibliográficas.}

Aliyah, Istijabatul; Setioko, Bambang y Pradoto, Wisnu (2017). Spatial flexibility in cultural mapping of traditional market areas in Surakarta (A case study of Pasar Gede in Surakata). City, Culture and Society, 10, 41-51. <DOI: https:// doi.org/10.1016/j.ccs.2017.05.004>

Axelsson, Robert; Angelstam, P., Degerman, E., Teitelbaum, S., Andersson, K., Elbakidze, M., y Drotz, M.K. (2013). Social and Cultural Sustainability: Criteria, Indicators, Verifier Variables for Measurement and Maps of Visualization to Support Planning. AMBIO 42(2), 215-228. <DOI: 10.1007/s13280=012-0376-0>

Bianchini, Franco; Parkinson, Michael (eds.) (1993). Cultural Policy and Urban Regeneration: The West European Experience. Manchester: Manchester University Press. <DOI: https:// doi.org/10.1080/00420989420080461>

Bianchini, Franco y Ghilardi, Lia (2007). Thinking culturally about place. Place Branding and Public Diplomacy, 3(4), 280-286. <DOI: https://doi.org/10.1057/palgrave.pb.6000077>

Chiesi, Leonardo y Costa, Paolo. (2015). One Strategy, Many Purposes: A Classification for Cultural Mapping Projects. In Cultural Mapping as cultural inquiry, (pp. 87-103). Routledge.

Contreras Gatica, Yasna (2011). La recuperación urbana y residencial del centro de Santiago: Nuevos habitantes, cambios socioespaciales significativos. Eure (Santiago), 37(112), 89113. <DOI: http://dx.doi.org/10.4067/S025071612011000300005>

Crawhall, Nigel (2007). The role of participatory cultural mapping in promoting intercultural dialogue- 'We are not hyenas'. Concept paper prepared for UNESCO Division of Cultural
Policies and Intercultural Dialogues.

Cuachi-Santoro, Roberta (2016). Mapping community identity: Safeguarding the memories of a city's downtown core. City, Culture and Society, 7(1), 43-54.

Chapin, F. Stuart y Knapp, Corrine N. (2015). Sense of place: A process for identifying and negotiating potentially contested visions of sustainability. Environmental Science \& Policy, 53, 38-46.

Daniel, Terry C.; Muhar, Andreas; Arnberger, Arne; Aznar, Olivier; Boyd, W. James; Chan, Kai M.A.; Elmqvist, Thomas; Flint, G. Courtney; Gobster, H. Paul; Grêt-Regamey, Adrienne; Lave, Rebecca; Muhar, Susanne; Penker, Marianne; Ribe G. Robert; Schauppenlehner, Thomas; Sikor, Thomas; Soloviy, Ihor; Spierenburg, Marja; Taczanowska, Karolina; Tam, Jordan y Von der Dunk, Andreas (2012). Contributions of cultural services to the ecosystem services agenda. Proceedings of the National Academy of Sciences, 109(23), 8812-8819.

Degen, Mónica y García, Marisol. (2012). The transformation of the 'Barcelona model': an analysis of culture, urban regeneration and governance. International journal of urban and regional research, 36(5), 1022-1038. <https:// doi.org/10.1111/j.1468-2427.2012.01152.x>.

Dinardi, Cecilia (2015). Unsettling the role of culture as panacea: The politics of cultureled urban regeneration in Buenos Aires. City, Culture and Society, 6(2), 9-18. <https://doi. org/10.1016/j.ccs.2015.03.003>.

Duque Franco, Isabel (2015). La cultura como estrategiade transformacióny promociónurbana en Bogotá y Medellín. Revista de Geografia Norte Grande, (61), 25-43. <http://dx.doi. org/10.4067/S0718-34022015000200003>.

Duxbury, Nancy. (2014). Cultural governance in sustainable cities.

Duxbury, Nancy; Hosagrahar, Jyoti y Pascual, Jordi (2016). Why must culture be at the heart of sustainable urban development? Agenda 21 for Culture.

Evans, Graeme y Shaw, Phyllida (2004). The contribution of culture to regeneration in the UK: a review of evidence. London: DCMS, 4.

Evans, Graeme (2005). Measure for measure: Evaluating the evidence of culture's contribution to regeneration. Urban Studies, 42(5-6), 959983. $<$ DOI: $10.1080=00420980500107102>$.

Freitas, Raquel (2016). Cultural mapping as a 
development tool. City, Culture and Society 7 (1), 9-16.

García, Beatriz (2004). Cultural policy and urban regeneration in Western European cities: lessons from experience, prospects for the future. Local economy, 19(4), 312-326. $<$ DOI: $10.1080 / 0269094042000286828>$.

Garza-Rodríguez, Fabiola (2015). Evaluación de indicadores socio-urbanos y estrategias de reforma para el centro de Monterrey. VII Seminario Internacional de Investigación en Urbanismoo, Barcelona-Montevideo, junio 2015. Departament d'Urbansime i Ordenació del Territori. Universitat Politècnica de Catalunya.

Hawkes, Jon (2001). The fourth pillar of sustainability: culture's essential role in public planning. Common Ground Publishing Pty Ltd in association with the Cultural Development Network (Vic).

Jeannotte, M. Sharon (2016). Story-telling about place: Engaging citizens in cultural mapping. City, Culture and Society, 7(1), 35-41

Kurjenoja, Anne Kristiina y Simental, María Emilia Ismael (2015). Ciudad como imagen: Xanenetla, Puebla, México, la "Ciudad Mural". Revista Internacional de Ciencias Humanas, 4(2).

Lazarević, Eva Vaništa; Koružnjak, Arch Boris y Devetaković, Mirjana (2016). Culture designled regeneration as a tool used to regenerate deprived areas. Belgrade-The Savamala quarter; reflections on an unplanned cultural zone. Energy and Buildings, 115, 3-10. <http:// dx.doi.org/10.1016/j.enbuild.2015.03.015>.

Landry, Charles y Bianchini, Franco (1995). The creative city. Londres: Demos. En http://www. demos.co.uk/files/thecreativecity.pdf

Landry, Charles (2003). Imagination and regeneration: Cultural policy and the future of cities. Cultural Policy and Action Department, Directorate General IV, Council of Europe.

Longley, Alys y Duxbury, Nancy (2016). Introduction: Mapping cultural intangibles. City, Culture and Society , 1 (7), 1-7. < https:// doi.org/10.1016/j.ccs.2015.12.006>

Martí-Costa, Marc y Pradel I Miquel, Marc (2011). The knowledge city against urban creativity? Artists' workshops and urban regeneration in Barcelona. European Urban and Regional Studies, 19(1), 92-108. <DOI: 10.1177/0969776411422481>.

Markusen, Ann (2007). The urban core as cultural sticky place. Time space places, 173-187.
Miles, Steven y Paddison, Ronan (2005). Introduction: The rise and rise of culture-led urban regeneration. 833-839.

Montgomery, John (2003). Cultural Quarters as Mechanisms for Urban Regeneration. Part 1: Conceptualising Cultural Quarters. Planning, Practice and Research, 18(4), 293-306. <https:// doi.org/10.1080/1561426042000215614>.

Moss, Linda (2002). Sheffield's cultural industries quarter 20 years on: What can be learned from a pioneering example? International Journal of Cultural Policy, 8(2), 211-219. <https://doi.org /10.1080/1028663022000009551>

Oktay, Derya (2012). Human sustainable urbanism: In pursuit of ecological and social-cultural sustainability. Procedia-Social and Behavioral Sciences, 36, 16-27. <https://doi.org/10.1016/j. sbspro.2012.03.003>

Pillai, Janet (2013). Cultural Mapping: A Guide to Understanding Place, Community, and Continuity. Strategic Information and Research Development Centre.

Rueda, Salvador; de Cáceres, Rafael; Cuchí, Albert y Brau, Luis. (2012). El urbanismo ecológico. Agencia de Ecología Urbana de Barcelona, Barcelona.

Soini, Katriina y Birkeland, Inger (2014). Exploring the scientific discourse on cultural sustainability. Geoforum, 51, 213-223. <https:// doi.org/10.1016/j.geoforum.2013.12.001>

Tavano Blessi, Giorgio; Tremblay, DianeGabrielle; Sandri, Marco; Pilati, Thomas (2012). New trajectories in urban regeneration processes: Cultural capital as source of human and social capital accumulation-Evidence from the case of Tohu in Montreal. Cities, 29(6), 397-407. <DOI: https://doi.org/10.1016/j. cities.2011.12.001>

Valdés Lange, Carlos (2018). La Arquitectura como Dispositivo de Regeneración Urbana: 20 años del Museo Guggenheim de Bilbao. Bitácora Urbano Territorial, 28(2), 115-123. <https:// doi.org/10.15446/bitacora.v28n2.70153>.

$\mathrm{Wu}, \mathrm{W}$. (2005). Dynamic cities and creative clusters. Washington DC, The World Bank.

Zarlenga, I. Matías; Ulldemolins, Rius Joaquim y Morató, Rodríguez Arturo. (2016). Cultural clusters and social interaction dynamics: The case of Barcelona. European Urban and Regional Studies, 23(3), 422-440.

Zukin, Sharon (1995). The Culture of Cities. Malden, 1995. 
Zukin, Sharon y Braslow, Laura (2011). The life cycle of New York's creative districts: Reflections on the unanticipated consequences of unplanned cultural zones. City, Culture and Society, 2(3), 131-140. <https://doi. org/10.1016/j.ccs.2011.06.003>. 